\title{
Bank Integration and Transmission of Financial Shocks: Evidence from Japan
}

\author{
Masami Imai, Seitaro Takarabe
}

Web Appendix

\section{Not for Publication}

Appendix B: Replicate Tables 1-7, using city bank deposit share as a measure of bank integration

Table B1: Replicate Table 1, using city bank deposit share as a measure of bank integration Columns 1-4 report the results of OLS. Panels A and B of columns 5-6 report the results of the first stage and second stage of IV regressions. $\triangle L O A N$ and $\triangle G D P$ represent growth of local loan and output, respectively. $\triangle$ Local Land Price is growth of local land price index and $\Delta$ City Land Price is growth of land price index for six major cities. City Bank Share is a ratio of city banks' deposit total deposit in a given prefecture. Standard errors are adjusted for clustering within each prefecture. First stage F-statistics is Kleibergen-Paap rk Wald F statistic (weak identification test).

\begin{tabular}{|c|c|c|c|c|c|c|}
\hline & (1) & (2) & (3) & (4) & (5) & (6) \\
\hline & \multicolumn{6}{|c|}{ Panel A } \\
\hline & \multicolumn{4}{|c|}{ OLS } & \multicolumn{2}{|c|}{ IV (1 $1^{\text {st }}$ stage $)$} \\
\hline Variables & $\Delta \mathrm{GDP}$ & $\Delta \mathrm{GDP}$ & $\Delta \mathrm{GDP}$ & $\triangle \mathrm{LOAN}$ & $\triangle \mathrm{LOAN}$ & $\triangle \mathrm{LOAN}$ \\
\hline City Bank Share ${ }^{*} \Delta$ City Land Price & & $\begin{array}{c}0.216 * * * \\
(0.0487)\end{array}$ & $\begin{array}{c}0.187 * * * \\
(0.0435)\end{array}$ & & $\begin{array}{c}0.613^{* * *} \\
(0.221)\end{array}$ & $\begin{array}{c}0.494 * * * \\
(0.182)\end{array}$ \\
\hline City Bank Share & & $\begin{array}{c}0.0775 * * \\
(0.0355)\end{array}$ & $\begin{array}{c}0.0739 * * \\
(0.0342)\end{array}$ & & $\begin{array}{c}0.144 \\
(0.0919)\end{array}$ & $\begin{array}{c}0.129 \\
(0.0850)\end{array}$ \\
\hline$\Delta$ Local Land Price & $\begin{array}{c}0.0222^{* * *} \\
(0.00648)\end{array}$ & & $\begin{array}{l}0.0129 * * \\
(0.00607)\end{array}$ & $\begin{array}{c}0.0781^{* * *} \\
(0.0167)\end{array}$ & & $\begin{array}{c}0.0534 * * * \\
(0.0134)\end{array}$ \\
\hline Observations & 920 & 916 & 916 & 920 & 916 & 916 \\
\hline Number of prefectures & 40 & 40 & 40 & 40 & 40 & 40 \\
\hline R-squared & 0.712 & 0.716 & 0.717 & 0.894 & 0.897 & 0.899 \\
\hline \multirow[t]{2}{*}{ First Stage F Statistic } & & & & & 7.691 & 7.336 \\
\hline & & & & & \multicolumn{2}{|c|}{$\begin{array}{c}\text { Panel B } \\
\operatorname{IV}\left(2^{\text {nd }} \text { stage }\right)\end{array}$} \\
\hline Variables & & & & & $\Delta \mathrm{GDP}$ & $\Delta \mathrm{GDP}$ \\
\hline$\triangle \mathrm{LOAN}$ & & & & & $\begin{array}{c}0.352^{* * *} \\
(0.0544)\end{array}$ & $\begin{array}{c}0.379 * * * \\
(0.0676)\end{array}$ \\
\hline$\Delta$ Local Land Price & & & & & & $\begin{array}{l}-0.00735 \\
(0.00761)\end{array}$ \\
\hline City Bank Share & & & & & $\begin{array}{c}0.0269 \\
(0.0359)\end{array}$ & $\begin{array}{c}0.0251 \\
(0.0374)\end{array}$ \\
\hline R-squared & & & & & 0.645 & 0.631 \\
\hline
\end{tabular}

Standard errors in parentheses 


\section{Not for Publication}

Table B2: Replicate Table 2, using city bank deposit share as a measure of bank integration

Columns 1-4, 5-8, and 9-12 report the results based on 2-year frequency, 3-year frequency, and 4-year frequency, respectively. Panels A and B in columns 5 and 6 report the results of the first stage and second stage of an IV regression. $\triangle L O A N$ and $\triangle G D P$ represent growth of local loan and output, respectively. $\triangle L o c a l$ Land Price is growth of local land price index and $\Delta$ City Land Price is growth of land price index for six major cities. City Bank Share is a ratio of city banks' deposit total deposit in a given prefecture.

Standard errors are adjusted for clustering within each prefecture. First stage F-statistics is Kleibergen-Paap rk Wald F statistic (weak identification test).

\begin{tabular}{|c|c|c|c|c|c|c|c|c|c|c|c|c|}
\hline & (1) & (2) & (3) & (4) & (5) & (6) & (7) & (8) & (9) & (10) & (11) & (12) \\
\hline & \multicolumn{12}{|c|}{ Panel A } \\
\hline & \multicolumn{4}{|c|}{ 2-Year Frequency } & \multicolumn{4}{|c|}{ 3-Year Frequency } & \multicolumn{4}{|c|}{ 4-Year Frequency } \\
\hline Variables & $\Delta \mathrm{GDP}$ & $\Delta \mathrm{GDP}$ & $\triangle \mathrm{LOAN}$ & $\triangle \mathrm{LOAN}$ & $\Delta \mathrm{GDP}$ & $\Delta \mathrm{GDP}$ & $\triangle \mathrm{LOAN}$ & $\triangle \mathrm{LOAN}$ & $\Delta \mathrm{GDP}$ & $\Delta \mathrm{GDP}$ & $\triangle \mathrm{LOAN}$ & $\triangle \mathrm{LOAN}$ \\
\hline$\Delta$ Local Land Price & $\begin{array}{l}0.0399 * * * \\
(0.00844)\end{array}$ & $\begin{array}{l}0.0276 * * * \\
(0.00866)\end{array}$ & $\begin{array}{l}0.120 * * * \\
(0.0203)\end{array}$ & $\begin{array}{l}0.0862 * * * \\
(0.0173)\end{array}$ & $\begin{array}{l}0.0448^{* * *} \\
(0.0104)\end{array}$ & $\begin{array}{l}0.0259 * * \\
(0.0109)\end{array}$ & $\begin{array}{l}0.147 * * * \\
(0.0316)\end{array}$ & $\begin{array}{l}0.0982^{* * *} \\
(0.0303)\end{array}$ & $\begin{array}{l}0.0566 * * * \\
(0.00965)\end{array}$ & $\begin{array}{l}0.0400 * * * \\
(0.0119)\end{array}$ & $\begin{array}{l}0.147 * * * \\
(0.0171)\end{array}$ & $\begin{array}{l}0.103 * * * \\
(0.0164)\end{array}$ \\
\hline $\begin{array}{l}\text { City Bank Share }{ }^{\star} \Delta \text { City } \\
\text { Land Price }\end{array}$ & & $\begin{array}{l}0.175^{* * *} \\
(0.0314)\end{array}$ & & $\begin{array}{l}0.478^{* * *} \\
(0.159)\end{array}$ & & $\begin{array}{l}0.190 * * * \\
(0.0369)\end{array}$ & & $\begin{array}{l}0.511^{* * *} \\
(0.183)\end{array}$ & & $\begin{array}{l}0.147^{* * *} \\
(0.0313)\end{array}$ & & $\begin{array}{l}0.386 * * * \\
(0.128)\end{array}$ \\
\hline City Bank Share & & $\begin{array}{l}0.0608 \\
(0.0433)\end{array}$ & & $\begin{array}{l}0.162 \\
(0.106)\end{array}$ & & $\begin{array}{l}0.403 \\
(0.358)\end{array}$ & & $\begin{array}{l}1.090 * * \\
(0.414)\end{array}$ & & $\begin{array}{l}0.0117 \\
(0.0564)\end{array}$ & & $\begin{array}{l}0.0204 \\
(0.130)\end{array}$ \\
\hline Observations & 440 & 438 & 440 & 438 & 280 & 278 & 280 & 278 & 200 & 199 & 200 & 199 \\
\hline Number of prefectures & 40 & 40 & 40 & 40 & 40 & 40 & 40 & 40 & 40 & 40 & 40 & 40 \\
\hline R-squared & 0.811 & 0.816 & 0.904 & 0.910 & 0.859 & 0.868 & 0.843 & 0.869 & 0.854 & 0.860 & 0.844 & 0.865 \\
\hline First Stage F Statistic & & & & 9.082 & & & & 7.833 & & & & 9.046 \\
\hline & & & & & & Panel B (IV & $2^{\text {nd }}$ stage) & & & & & \\
\hline & & & & $\Delta \mathrm{GDP}$ & & & & $\Delta \mathrm{GDP}$ & & & & $\Delta \mathrm{GDP}$ \\
\hline$\triangle \mathrm{LOAN}$ & & & & $\begin{array}{l}0.366^{* * * *} \\
(0.0859)\end{array}$ & & & & $\begin{array}{l}0.372^{* * *} \\
(0.111)\end{array}$ & & & & $\begin{array}{l}0.380 * * * \\
(0.143)\end{array}$ \\
\hline$\Delta$ Local Land Price & & & & $\begin{array}{l}-0.00390 \\
(0.0135)\end{array}$ & & & & $\begin{array}{l}-0.0106 \\
(0.0205)\end{array}$ & & & & $\begin{array}{l}0.000949 \\
(0.0248)\end{array}$ \\
\hline City Bank Share & & & & $\begin{array}{l}0.00152 \\
(0.0300)\end{array}$ & & & & $\begin{array}{l}-0.00278 \\
(0.279)\end{array}$ & & & & $\begin{array}{l}0.00392 \\
(0.0452)\end{array}$ \\
\hline R-squared & & & & 0.751 & & & & 0.844 & & & & 0.835 \\
\hline
\end{tabular}

Standard errors in parentheses 


\section{Not for Publication}

Table B3: Replicate Table 3, using city bank deposit share as a measure of bank integration

The reported results are based upon the value of city bank share prevailing as of 1979, which is kept constant for the entire sample period. $\triangle L O A N$ and $\triangle G D P$ represent growth of local loan and output, respectively. $\Delta$ Local Land Price is growth of local land price index and $\Delta$ City Land Price is growth of land price index for six major cities. City Bank Share is a ratio of city banks' deposit total deposit in a given prefecture. Standard errors are adjusted for clustering within each prefecture. First stage F-statistics is Kleibergen-Paap rk Wald F statistic (weak identification test).

\begin{tabular}{|c|c|c|}
\hline & (1) & (2) \\
\hline & \multicolumn{2}{|c|}{ Panel A } \\
\hline & OLS & IV $\left(1^{\text {st }}\right.$ stage $)$ \\
\hline & $\Delta \mathrm{GDP}$ & $\triangle \mathrm{LOAN}$ \\
\hline \multirow[t]{2}{*}{$\Delta$ Local Land Price } & $0.0123^{*}$ & $0.0547 * * *$ \\
\hline & $(0.00629)$ & $(0.0140)$ \\
\hline \multirow[t]{2}{*}{ City Bank Share* $\Delta$ City Land Price (1979) } & $0.186 * * *$ & $0.486 * *$ \\
\hline & $(0.0421)$ & $(0.189)$ \\
\hline Observations & 897 & 897 \\
\hline Number of prefectures & 39 & 39 \\
\hline \multirow[t]{5}{*}{ R-squared } & 0.719 & 0.915 \\
\hline & & 6.588 \\
\hline & & Panel B \\
\hline & & IV ( $2^{\text {nd }}$ stage $)$ \\
\hline & & $\Delta \mathrm{GDP}$ \\
\hline \multirow[t]{2}{*}{$\triangle \mathrm{LOAN}$} & & $0.383 * * *$ \\
\hline & & $(0.0793)$ \\
\hline \multirow[t]{2}{*}{$\Delta$ Local Land Price } & & -0.00861 \\
\hline & & $(0.00849)$ \\
\hline R-squared & & 0.618 \\
\hline
\end{tabular}

Standard errors in parentheses 


\section{Not for Publication}

Table B4: Replicate Table 4, using city bank deposit share as a measure of bank integration

The table reports the results of the specification that separately estimate the effects of city land price growth on non-city bank lending (columns 1 and 2 ) and city bank lending (columns 3 and 4). Panels $\mathrm{A}$ and B in column 4 report the results of the first stage and second stage of an IV regression, respectively. $\triangle L O A N$ and $\triangle G D P$ represent growth of local loan and output, respectively. $\Delta$ Local Land Price is growth of local land price index and $\Delta C i t y$ Land Price is growth of land price index for six major cities. City Bank Share is a ratio of city banks' deposit total deposit in a given prefecture. Standard errors are adjusted for clustering within each prefecture. First stage F-statistics is Kleibergen-Paap rk Wald F statistic (weak identification test).

\begin{tabular}{|c|c|c|c|c|}
\hline & $(1)$ & $(2)$ & (3) & (4) \\
\hline & \multicolumn{4}{|c|}{ Panel A } \\
\hline & \multicolumn{2}{|c|}{$\triangle \mathrm{LOAN}$ (Non-City Bank) } & \multicolumn{2}{|c|}{$\Delta \mathrm{LOAN}($ City Bank) } \\
\hline$\Delta$ Local Land Price & $\begin{array}{c}0.0766 * * * \\
(0.0162)\end{array}$ & $\begin{array}{c}0.0630 * * * \\
(0.0158)\end{array}$ & $\begin{array}{c}0.0148 * * * \\
(0.00518)\end{array}$ & $\begin{array}{c}-0.00170 \\
(0.00266)\end{array}$ \\
\hline City Bank Share ${ }^{*} \Delta$ City Land Price & & $\begin{array}{c}0.276 \\
(0.180)\end{array}$ & & $\begin{array}{c}0.336 * * * \\
(0.0260)\end{array}$ \\
\hline City Bank Share & & $\begin{array}{c}0.155 \\
(0.119)\end{array}$ & & $\begin{array}{c}0.0131 \\
(0.0326)\end{array}$ \\
\hline Observations & 916 & 916 & 916 & 916 \\
\hline Number of prefectures & 40 & 40 & 40 & 40 \\
\hline R-squared & 0.889 & 0.890 & 0.287 & 0.519 \\
\hline First Stage F Statistic & & & & 166.6 \\
\hline & & & & $\begin{array}{c}\text { Panel B } \\
\left(\mathrm{IV}, 2^{\text {nd }} \text { stage }\right) \\
\Delta \mathrm{GDP}\end{array}$ \\
\hline$\triangle \mathrm{LOAN}$ (City Banks) & & & & $\begin{array}{c}0.557 * * * \\
(0.0905)\end{array}$ \\
\hline$\Delta$ Local Land Price & & & & $\begin{array}{l}0.0138 * * \\
(0.00628)\end{array}$ \\
\hline City Bank Share & & & & $\begin{array}{c}0.0666 * * \\
(0.0329)\end{array}$ \\
\hline R-squared & & & & 0.708 \\
\hline
\end{tabular}

Standard errors in parentheses 


\section{Not for Publication}

Table B5: Replicate Table 5, using city bank deposit share as a measure of bank integration

The table shows the results of specifications that control for differential correlation between local economies and cities. We compute the difference in income per capita (in absolute value) between each prefecture and cities (Income Diff) and then interact it to city land price growth and to city bank deposit share (columns 1-3). We interact the average distance to the cities (Distance) to city land price growth and to city bank deposit share (columns 4-6). We also compute industry mix control (columns 7-9), which is constructed as: $\eta_{i t}=\gamma_{i j t-1} \eta_{j t}$ where $\eta_{i t}$ denotes the predicted output growth of prefecture $i$ in year $t$, $\gamma_{j i t-1}$ denote the (lagged) share of output in industry $j$ in prefecture $i$ in year $t-1$, and $\eta_{j t}$ denote the output growth of industry $\mathrm{j}$ in city prefectures in year $t$. Standard errors are adjusted for clustering within each

\begin{tabular}{|c|c|c|c|c|c|c|c|c|c|}
\hline & (1) & (2) & (3) & (4) & (5) & (6) & (7) & (8) & (9) \\
\hline & $\begin{array}{l}\text { Reduced- } \\
\text { form } \\
\Delta \mathrm{GDP}\end{array}$ & $\begin{array}{c}\text { IV } \\
\text { (2nd stage) } \\
\Delta \text { GDP }\end{array}$ & $\begin{array}{c}\text { IV } \\
\text { (1st stage) } \\
\Delta \text { LOAN }\end{array}$ & $\begin{array}{l}\text { Reduced- } \\
\text { form } \\
\Delta \mathrm{GDP}\end{array}$ & $\begin{array}{c}\text { IV } \\
\text { (2nd stage) } \\
\Delta \text { GDP }\end{array}$ & $\begin{array}{c}\text { IV } \\
\text { (1st stage) } \\
\Delta \text { LOAN }\end{array}$ & $\begin{array}{l}\text { Reduced- } \\
\text { form } \\
\Delta \mathrm{GDP}\end{array}$ & $\begin{array}{c}\text { IV } \\
\text { (2nd stage) } \\
\Delta \mathrm{GDP}\end{array}$ & $\begin{array}{c}\text { IV } \\
\text { (1st stage) } \\
\Delta \text { LOAN }\end{array}$ \\
\hline City Bank Share* $\Delta$ City Land Price & $\begin{array}{c}0.133 * * * \\
(0.0280)\end{array}$ & & $\begin{array}{c}0.619 * * * \\
(0.193)\end{array}$ & $\begin{array}{c}0.110^{* * *} \\
(0.0290)\end{array}$ & & $\begin{array}{c}0.596 * * * \\
(0.196)\end{array}$ & $\begin{array}{c}0.0995 * * * \\
(0.0292)\end{array}$ & & $\begin{array}{c}0.595 * * * \\
(0.197)\end{array}$ \\
\hline$\triangle \mathrm{LOAN}$ & & $\begin{array}{c}0.215^{* * *} \\
(0.0761)\end{array}$ & & & $\begin{array}{c}0.184 * * * \\
(0.0698)\end{array}$ & & & $\begin{array}{l}0.167 * * \\
(0.0663)\end{array}$ & \\
\hline$\Delta$ Local Land Price & $\begin{array}{c}0.00790 \\
(0.00577)\end{array}$ & $\begin{array}{l}-0.00135 \\
(0.00693)\end{array}$ & $\begin{array}{c}0.0430 * * * \\
(0.0125)\end{array}$ & $\begin{array}{c}0.00881 \\
(0.00576)\end{array}$ & $\begin{array}{c}0.000765 \\
(0.00678)\end{array}$ & $\begin{array}{c}0.0438 * * * \\
(0.0126)\end{array}$ & $\begin{array}{l}0.00943 * \\
(0.00559)\end{array}$ & $\begin{array}{c}0.00210 \\
(0.00655)\end{array}$ & $\begin{array}{c}0.0438 * * * \\
(0.0126)\end{array}$ \\
\hline City Bank Share & $\begin{array}{l}0.173 * * \\
(0.0648)\end{array}$ & $\begin{array}{c}0.171^{* * *} \\
(0.0593)\end{array}$ & $\begin{array}{c}0.0100 \\
(0.0652)\end{array}$ & $\begin{array}{c}0.516^{* *} \\
(0.231)\end{array}$ & $\begin{array}{c}0.469 * * \\
(0.204)\end{array}$ & $\begin{array}{c}0.253 \\
(0.298)\end{array}$ & $\begin{array}{c}0.470 * * \\
(0.223)\end{array}$ & $\begin{array}{c}0.429 * * \\
(0.197)\end{array}$ & $\begin{array}{c}0.250 \\
(0.298)\end{array}$ \\
\hline Income Diff* $\Delta$ City Land Price & $\begin{array}{c}-0.0329 * * * \\
(0.00639)\end{array}$ & $\begin{array}{c}-0.0183^{* *} \\
(0.00806)\end{array}$ & $\begin{array}{c}-0.0677 * * * \\
(0.0175)\end{array}$ & $\begin{array}{c}-0.0274 * * * \\
(0.00890)\end{array}$ & $\begin{array}{c}-0.0161 * \\
(0.00912)\end{array}$ & $\begin{array}{c}-0.0615^{* * *} \\
(0.0218)\end{array}$ & $\begin{array}{c}-0.0237 * * * \\
(0.00868)\end{array}$ & $\begin{array}{c}-0.0134 \\
(0.00880)\end{array}$ & $\begin{array}{c}-0.0612^{* * *} \\
(0.0217)\end{array}$ \\
\hline Income Diff*City Bank Share & $\begin{array}{l}-0.104 * \\
(0.0520)\end{array}$ & $\begin{array}{l}-0.122 * * \\
(0.0485)\end{array}$ & $\begin{array}{c}0.0845 * * \\
(0.0370)\end{array}$ & $\begin{array}{l}-0.120 * * \\
(0.0575)\end{array}$ & $\begin{array}{l}-0.133^{* *} \\
(0.0531)\end{array}$ & $\begin{array}{c}0.0744 * \\
(0.0422)\end{array}$ & $\begin{array}{l}-0.117 * * \\
(0.0579)\end{array}$ & $\begin{array}{l}-0.130 * * \\
(0.0536)\end{array}$ & $\begin{array}{l}0.0746 * \\
(0.0422)\end{array}$ \\
\hline Distance $* \Delta$ City Land Price & & & & $\begin{array}{l}-0.00912 \\
(0.0144)\end{array}$ & $\begin{array}{c}-0.00670 \\
(0.0131)\end{array}$ & $\begin{array}{c}-0.0131 \\
(0.0441)\end{array}$ & $\begin{array}{l}-0.00991 \\
(0.0135)\end{array}$ & $\begin{array}{l}-0.00770 \\
(0.0128)\end{array}$ & $\begin{array}{l}-0.0132 \\
(0.0441)\end{array}$ \\
\hline Distance*City Bank Share & & & & $\begin{array}{l}-1.016 \\
(0.632)\end{array}$ & $\begin{array}{l}-0.883 \\
(0.575)\end{array}$ & $\begin{array}{l}-0.724 \\
(0.774)\end{array}$ & $\begin{array}{l}-0.902 \\
(0.609)\end{array}$ & $\begin{array}{l}-0.783 \\
(0.554)\end{array}$ & $\begin{array}{l}-0.714 \\
(0.774)\end{array}$ \\
\hline Industry Mix Control $\left(\eta_{i t}\right)$ & & & & & & & $\begin{array}{c}0.566 * * \\
(0.232)\end{array}$ & $\begin{array}{c}0.558 * * \\
(0.226)\end{array}$ & $\begin{array}{l}0.0461 \\
(0.217)\end{array}$ \\
\hline Observations & 916 & 916 & 916 & 916 & 916 & 916 & 916 & 916 & 916 \\
\hline R-squared & 0.723 & 0.703 & 0.902 & 0.725 & 0.712 & 0.902 & 0.728 & 0.718 & 0.902 \\
\hline Number of prefectures & 40 & 40 & 40 & 40 & 40 & 40 & 40 & 40 & 40 \\
\hline First Stage F Statistic & & 10.26 & & & 9.236 & & & 9.152 & \\
\hline
\end{tabular}

*** $\mathrm{p}<0.01, * * \mathrm{p}<0.05,{ }^{*} \mathrm{p}<0.1$

Standard errors in parentheses 


\section{Not for Publication}

Table B6: Replicate Table 6, using city bank deposit share as a measure of bank

Columns 1-2, 3-4, and 5-6 show the results based on a subset of prefectures whose distance to the cities is more than 50, 100, and 150 kilometers, respectively. Columns 7-8, 9-10, and 11-12 show the results based on prefectures whose income per capita differ from income per capita in the cities by more than 0.6, 0.8 , and one million yen, respectively. The reported correlation coefficient at the bottom of the table is the average correlation coefficient between city land price growth and local land price growth in the excluded and included prefectures. Standard errors are adjusted for clustering within each prefecture. First stage Fstatistics is Kleibergen-Paap rk Wald F statistic (weak identification test).

\begin{tabular}{|c|c|c|c|c|c|c|c|c|c|c|c|c|}
\hline & (1) & (2) & (3) & (4) & (5) & (6) & (7) & (8) & (9) & (10) & (11) & (12) \\
\hline & \multicolumn{2}{|c|}{ Dist $>50 \mathrm{~km}$} & \multicolumn{2}{|c|}{ Dist $>100 \mathrm{~km}$} & \multicolumn{2}{|c|}{ Dist $>150 \mathrm{~km}$} & \multicolumn{2}{|c|}{$\begin{array}{l}\text { Income Diff }> \\
0.6 \text { million yen }\end{array}$} & \multicolumn{2}{|c|}{$\begin{array}{c}\text { Income Diff }> \\
0.8 \text { million yen }\end{array}$} & \multicolumn{2}{|c|}{$\begin{array}{c}\text { Income Diff> } \\
\text { one million yen }\end{array}$} \\
\hline & $\Delta \mathrm{GDP}$ & $\triangle \mathrm{LOAN}$ & $\Delta \mathrm{GDP}$ & $\triangle \mathrm{LOAN}$ & $\Delta \mathrm{GDP}$ & $\triangle \mathrm{LOAN}$ & $\Delta \mathrm{GDP}$ & $\triangle \mathrm{LOAN}$ & $\Delta \mathrm{GDP}$ & $\triangle \mathrm{LOAN}$ & $\Delta \mathrm{GDP}$ & $\triangle \mathrm{LOAN}$ \\
\hline$\Delta$ Local Land Price & $\begin{array}{c}0.00979 \\
(0.00770)\end{array}$ & $\begin{array}{c}0.0279 * * \\
(0.0124)\end{array}$ & $\begin{array}{c}0.0158 * \\
(0.00812)\end{array}$ & $\begin{array}{c}0.0267 * \\
(0.0143)\end{array}$ & $\begin{array}{c}0.0127 * \\
(0.00710)\end{array}$ & $\begin{array}{c}0.0275^{*} \\
(0.0146)\end{array}$ & $\begin{array}{c}0.0109 \\
(0.00659)\end{array}$ & $\begin{array}{c}0.0499 * * * \\
(0.0135)\end{array}$ & $\begin{array}{c}0.0136 * \\
(0.00707)\end{array}$ & $\begin{array}{c}0.0516^{* * *} \\
(0.0149)\end{array}$ & $\begin{array}{l}0.0128 * * \\
(0.00592)\end{array}$ & $\begin{array}{c}0.0538 * * * \\
(0.0167)\end{array}$ \\
\hline $\begin{array}{l}\text { City Bank Share } * \Delta \text { City Land } \\
\text { Price }\end{array}$ & $\begin{array}{c}0.367 * * \\
(0.170)\end{array}$ & $\begin{array}{c}1.773 * * * \\
(0.316)\end{array}$ & $\begin{array}{l}0.303^{*} \\
(0.176)\end{array}$ & $\begin{array}{c}1.502^{* * *} \\
(0.338)\end{array}$ & $\begin{array}{l}0.348 * * \\
(0.152)\end{array}$ & $\begin{array}{c}1.276^{* * * *} \\
(0.420)\end{array}$ & $\begin{array}{l}0.197 * * * \\
(0.0456)\end{array}$ & $\begin{array}{c}0.497 * * * \\
(0.173)\end{array}$ & $\begin{array}{l}0.200^{* * *} \\
(0.0450)\end{array}$ & $\begin{array}{c}0.505^{* * *} \\
(0.180)\end{array}$ & $\begin{array}{l}0.203 * * * \\
(0.0364)\end{array}$ & $\begin{array}{c}0.501^{* * *} \\
(0.149)\end{array}$ \\
\hline City Bank Share & $\begin{array}{l}0.121 * * \\
(0.0452)\end{array}$ & $\begin{array}{c}0.231^{* * *} \\
(0.0479)\end{array}$ & $\begin{array}{l}0.0780 * \\
(0.0391)\end{array}$ & $\begin{array}{c}0.167 * * * \\
(0.0463)\end{array}$ & $\begin{array}{l}-0.0980 \\
(0.289)\end{array}$ & $\begin{array}{c}0.343 \\
(0.329)\end{array}$ & $\begin{array}{c}0.0759 * * \\
(0.0361)\end{array}$ & $\begin{array}{c}0.102 \\
(0.0753)\end{array}$ & $\begin{array}{l}0.0935 \\
(0.132)\end{array}$ & $\begin{array}{l}0.305^{*} \\
(0.179)\end{array}$ & $\begin{array}{c}0.0140 \\
(0.0528)\end{array}$ & $\begin{array}{l}0.0371 \\
(0.161)\end{array}$ \\
\hline Observations & 801 & 801 & 663 & 663 & 525 & 525 & 801 & 801 & 663 & 663 & 479 & 479 \\
\hline Number of prefectures & 35 & 35 & 29 & 29 & 23 & 23 & 35 & 35 & 29 & 29 & 21 & 21 \\
\hline R-squared & 0.698 & $\begin{array}{c}0.913 \\
\text { IV } \\
2^{\text {nd }} \text { stage } \\
\text { GDP }\end{array}$ & 0.725 & $\begin{array}{c}0.912 \\
\text { IV } \\
2^{\text {nd }} \text { stage } \\
\text { GDP }\end{array}$ & 0.738 & $\begin{array}{c}0.912 \\
\text { IV } \\
2^{\text {nd }} \text { stage } \\
\text { GDP }\end{array}$ & 0.708 & $\begin{array}{c}0.896 \\
\text { IV } \\
2^{\text {nd }} \text { stage } \\
\text { GDP }\end{array}$ & 0.711 & $\begin{array}{c}0.887 \\
\text { IV } \\
2^{\text {nd }} \text { stage } \\
\text { GDP }\end{array}$ & 0.728 & $\begin{array}{c}0.867 \\
\text { IV } \\
2^{\text {nd }} \text { stage } \\
\text { GDP }\end{array}$ \\
\hline$\triangle \mathrm{LOAN}$ & & $\begin{array}{c}0.207 * * * \\
(0.0801)\end{array}$ & & $\begin{array}{l}0.202^{* *} \\
(0.0953)\end{array}$ & & $\begin{array}{c}0.273 * * \\
(0.137)\end{array}$ & & $\begin{array}{c}0.397 * * * \\
(0.0648)\end{array}$ & & $\begin{array}{c}0.396 * * * \\
(0.0721)\end{array}$ & & $\begin{array}{c}0.405^{* * * *} \\
(0.0671)\end{array}$ \\
\hline$\Delta$ Local Land Price & & $\begin{array}{c}0.00400 \\
(0.00844)\end{array}$ & & $\begin{array}{c}0.0104 \\
(0.00856)\end{array}$ & & $\begin{array}{c}0.00516 \\
(0.00802)\end{array}$ & & $\begin{array}{c}-0.00892 \\
(0.00851)\end{array}$ & & $\begin{array}{l}-0.00681 \\
(0.00946)\end{array}$ & & $\begin{array}{l}-0.00896 \\
(0.00982)\end{array}$ \\
\hline City Bank Share & & $\begin{array}{c}0.0727 * * \\
(0.0327)\end{array}$ & & $\begin{array}{c}0.0443^{* *} \\
(0.0198)\end{array}$ & & $\begin{array}{l}-0.192 \\
(0.294)\end{array}$ & & $\begin{array}{c}0.0356 \\
(0.0335)\end{array}$ & & $\begin{array}{l}-0.0274 \\
(0.105)\end{array}$ & & $\begin{array}{l}-0.00104 \\
(0.0835)\end{array}$ \\
\hline $\begin{array}{l}\text { R-squared } \\
\text { First Stage F Statistic }\end{array}$ & & $\begin{array}{l}0.683 \\
31.51\end{array}$ & & $\begin{array}{l}0.706 \\
19.72\end{array}$ & & $\begin{array}{l}0.693 \\
9.214\end{array}$ & & $\begin{array}{l}0.599 \\
8.265\end{array}$ & & $\begin{array}{l}0.580 \\
7.855\end{array}$ & & $\begin{array}{l}0.543 \\
11.33\end{array}$ \\
\hline $\begin{array}{l}\text { Corr. Coef. (Excluded } \\
\text { Prefectures) }\end{array}$ & \multicolumn{2}{|c|}{.82} & \multicolumn{2}{|c|}{.69} & \multicolumn{2}{|c|}{.66} & \multicolumn{2}{|c|}{.77} & \multicolumn{2}{|c|}{.67} & \multicolumn{2}{|c|}{.63} \\
\hline $\begin{array}{l}\text { Corr. Coef. (Included } \\
\text { Prefectures) }\end{array}$ & \multicolumn{2}{|c|}{.55} & \multicolumn{2}{|c|}{.54} & \multicolumn{2}{|c|}{.53} & \multicolumn{2}{|c|}{.55} & \multicolumn{2}{|c|}{.55} & \multicolumn{2}{|c|}{.54} \\
\hline
\end{tabular}

Standard errors in parentheses 
Not for Publication

Table B7: Replicate Table 7, using city bank deposit share as a measure of bank

Columns 1-2 report the results of panel corrected standard errors (Beck and Katz, 1995). Columns 3-4 report the results of Driscoll-Kraay standard errors (Driscoll and Kraay, 1998). Columns 3-4 report the results of two-way clustering (Petersen, 2009; Cameron, Gelbach, and Miller, 2006).

\begin{tabular}{|c|c|c|c|c|c|c|}
\hline & (1) & $\overline{(2)}$ & (3) & $(4)$ & (5) & (6) \\
\hline & \multicolumn{2}{|c|}{$\begin{array}{l}\text { Panel-Corrected Standard } \\
\text { Errors }\end{array}$} & \multicolumn{2}{|c|}{$\begin{array}{l}\text { Driscoll-Kraay Standard } \\
\text { Errors }\end{array}$} & \multicolumn{2}{|c|}{$\begin{array}{l}\text { Petersen-Cameron-Gelbach- } \\
\text { Miller Two-way Clustering }\end{array}$} \\
\hline & $\triangle \mathrm{LOAN}$ & $\Delta \mathrm{GDP}$ & $\triangle \mathrm{LOAN}$ & $\Delta \mathrm{GDP}$ & $\triangle \mathrm{LOAN}$ & $\Delta \mathrm{GDP}$ \\
\hline City Bank Share* $\Delta$ City Land Price & $\begin{array}{c}0.595^{* * *} \\
(0.216)\end{array}$ & $\begin{array}{c}0.0995^{* *} \\
(0.0497)\end{array}$ & $\begin{array}{c}0.595^{* * *} \\
(0.170)\end{array}$ & $\begin{array}{c}0.0995^{* *} \\
(0.0462)\end{array}$ & $\begin{array}{c}0.595^{* * *} \\
(0.201)\end{array}$ & $\begin{array}{c}0.0995 * * * \\
(0.0298)\end{array}$ \\
\hline$\Delta$ Local Land Price & $\begin{array}{l}0.0438 * * \\
(0.0174)\end{array}$ & $\begin{array}{c}0.00943 \\
(0.00693)\end{array}$ & $\begin{array}{c}0.0438 * * * \\
(0.0121)\end{array}$ & $\begin{array}{l}0.00943 * \\
(0.00539)\end{array}$ & $\begin{array}{c}0.0438 * * * \\
(0.0129)\end{array}$ & $\begin{array}{c}0.00943 \\
(0.00571)\end{array}$ \\
\hline City Bank Share & $\begin{array}{l}0.250 \\
(0.582)\end{array}$ & $\begin{array}{l}0.470 * * \\
(0.190)\end{array}$ & $\begin{array}{l}0.250 \\
(0.283)\end{array}$ & $\begin{array}{l}0.470 * * \\
(0.205)\end{array}$ & $\begin{array}{c}0.250 \\
(0.305)\end{array}$ & $\begin{array}{l}0.470 * * \\
(0.228)\end{array}$ \\
\hline Income Diff* $\Delta$ City Land Price & $\begin{array}{c}-0.0612^{* * *} \\
(0.0204)\end{array}$ & $\begin{array}{c}-0.0237 * * \\
(0.0119)\end{array}$ & $\begin{array}{c}-0.0612^{* * *} \\
(0.0147)\end{array}$ & $\begin{array}{l}-0.0237^{*} \\
(0.0120)\end{array}$ & $\begin{array}{c}-0.0612^{* * * *} \\
(0.0222)\end{array}$ & $\begin{array}{l}-0.0237 * * \\
(0.00888)\end{array}$ \\
\hline Income Diff*City Bank Share & $\begin{array}{l}0.0746 \\
(0.161)\end{array}$ & $\begin{array}{c}-0.117 * * * \\
(0.0317)\end{array}$ & $\begin{array}{c}0.0746 \\
(0.0613)\end{array}$ & $\begin{array}{l}-0.117 * * \\
(0.0440)\end{array}$ & $\begin{array}{l}0.0746 * \\
(0.0431)\end{array}$ & $\begin{array}{l}-0.117^{*} \\
(0.0592)\end{array}$ \\
\hline Distance $* \Delta$ City GDP & $\begin{array}{l}-0.0132 \\
(0.0264)\end{array}$ & $\begin{array}{l}-0.00991 \\
(0.0196)\end{array}$ & $\begin{array}{l}-0.0132 \\
(0.0140)\end{array}$ & $\begin{array}{r}-0.00991 \\
(0.0138)\end{array}$ & $\begin{array}{l}-0.0132 \\
(0.0451)\end{array}$ & $\begin{array}{l}-0.00991 \\
(0.0138)\end{array}$ \\
\hline Distance*City Bank Share & $\begin{array}{l}-0.714 \\
(1.451)\end{array}$ & $\begin{array}{l}-0.902 * \\
(0.536)\end{array}$ & $\begin{array}{l}-0.714 \\
(0.690)\end{array}$ & $\begin{array}{l}-0.902 \\
(0.580)\end{array}$ & $\begin{array}{l}-0.714 \\
(0.792)\end{array}$ & $\begin{array}{l}-0.902 \\
(0.623)\end{array}$ \\
\hline Industry Mix Control $\left(\eta_{i t}\right)$ & $\begin{array}{l}0.0461 \\
(0.474)\end{array}$ & $\begin{array}{l}0.566 * * \\
(0.253)\end{array}$ & $\begin{array}{l}0.0461 \\
(0.441)\end{array}$ & $\begin{array}{c}0.566 \\
(0.425)\end{array}$ & $\begin{array}{l}0.0461 \\
(0.222)\end{array}$ & $\begin{array}{c}0.566 * * \\
(0.237)\end{array}$ \\
\hline Observations & 916 & 916 & 916 & 916 & 916 & 916 \\
\hline Number of prefectures & 40 & 40 & 40 & 40 & 40 & 40 \\
\hline R-squared & 0.903 & 0.732 & 0.903 & 0.732 & 0.903 & 0.732 \\
\hline
\end{tabular}

$* * * \mathrm{p}<0.01, * * \mathrm{p}<0.05, * \mathrm{p}<0.1$

Standard errors in parentheses 


\section{Not for Publication}

Appendix C: Replicate Tables 1-7, using all prefectures (including the six city prefectures and Hokkaido)

\section{Table C1: Replicate Table 1, using all prefectures (including the six city prefectures and Hokkaido)}

Columns 1-4 report the results of OLS. Panels A and B of columns 5-6 report the results of the first stage and second stage of IV regressions. $\triangle L O A N$ and $\triangle G D P$ represent growth of local loan and output, respectively. $\Delta$ Local Land Price is growth of local land price index and $\Delta$ City Land Price is growth of land price index for six major cities. City Bank Share is a ratio of the number of city bank branches to the total number of bank branches in a given prefecture.

Standard errors are adjusted for clustering within each prefecture. First stage F-statistics is Kleibergen-Paap rk Wald F statistic (weak identification test).

\begin{tabular}{|c|c|c|c|c|c|c|}
\hline & (1) & $(2)$ & (3) & (4) & (5) & (6) \\
\hline & \multicolumn{6}{|c|}{ Panel A } \\
\hline & \multicolumn{4}{|c|}{ OLS } & \multicolumn{2}{|c|}{ IV ( $1^{\text {st }}$ stage $)$} \\
\hline Variables & $\Delta \mathrm{GDP}$ & $\Delta \mathrm{GDP}$ & $\Delta \mathrm{GDP}$ & $\triangle \mathrm{LOAN}$ & $\triangle \mathrm{LOAN}$ & $\triangle \mathrm{LOAN}$ \\
\hline City Bank Share $* \Delta$ City Land Price & & $\begin{array}{c}0.113 * * * \\
(0.0120)\end{array}$ & $\begin{array}{c}0.102 * * * \\
(0.0117)\end{array}$ & & $\begin{array}{c}0.315^{* * *} \\
(0.0494)\end{array}$ & $\begin{array}{c}0.269 * * * \\
(0.0556)\end{array}$ \\
\hline City Bank Share & & $\begin{array}{c}0.0165 \\
(0.0240)\end{array}$ & $\begin{array}{c}0.0186 \\
(0.0239)\end{array}$ & & $\begin{array}{c}0.0152 \\
(0.0761)\end{array}$ & $\begin{array}{c}0.0237 \\
(0.0768)\end{array}$ \\
\hline$\Delta$ Local Land Price & $\begin{array}{l}0.0142^{* *} \\
(0.00606)\end{array}$ & & $\begin{array}{c}0.00818 * * \\
(0.00387)\end{array}$ & $\begin{array}{c}0.0492 * * \\
(0.0222)\end{array}$ & & $\begin{array}{l}0.0333^{*} \\
(0.0175)\end{array}$ \\
\hline Observations & 1081 & 1081 & 1081 & 1081 & 1081 & 1081 \\
\hline Number of prefectures & 47 & 47 & 47 & 47 & 47 & 47 \\
\hline R-squared & 0.716 & 0.723 & 0.724 & 0.869 & 0.873 & 0.875 \\
\hline First Stage F Statistic & & & & & 40.71 & 23.47 \\
\hline & & & & & \multicolumn{2}{|c|}{$\begin{array}{c}\text { Panel B } \\
\operatorname{IV}\left(2^{\text {nd }} \text { stage }\right)\end{array}$} \\
\hline Variables & & & & & $\Delta \mathrm{GDP}$ & $\Delta \mathrm{GDP}$ \\
\hline$\triangle \mathrm{LOAN}$ & & & & & $\begin{array}{c}0.359 * * * \\
(0.0487)\end{array}$ & $\begin{array}{c}0.378^{* * *} \\
(0.0655)\end{array}$ \\
\hline$\Delta$ Local Land Price & & & & & & $\begin{array}{l}-0.00440 \\
(0.00550)\end{array}$ \\
\hline City Bank Share & & & & & $\begin{array}{c}0.0111 \\
(0.0185)\end{array}$ & $\begin{array}{c}0.00967 \\
(0.0193)\end{array}$ \\
\hline R-squared & & & & & 0.636 & 0.625 \\
\hline
\end{tabular}

Standard errors in parentheses 


\section{Not for Publication}

\section{Table C2: Replicate Table 2, using all prefectures (including the six city prefectures and Hokkaido)}

Columns 1-4, 5-8, and 9-12 report the results based on 2-year frequency, 3-year frequency, and 4-year frequency, respectively. Panels A and B in columns 5 and 6 report the results of the first stage and second stage of an IV regression. $\triangle L O A N$ and $\triangle G D P$ represent growth of local loan and output, respectively. $\Delta L o c a l$ Land Price is growth of local land price index and $\Delta$ City Land Price is growth of land price index for six major cities. City Bank Share is a ratio of the number of city bank branches to the total number of bank branches in a given prefecture. Standard errors are adjusted for clustering within each prefecture. First stage F-statistics is Kleibergen-Paap rk Wald F statistic (weak identification test).

\begin{tabular}{|c|c|c|c|c|c|c|c|c|c|c|c|c|}
\hline & $(1)$ & $(2)$ & (3) & (4) & (5) & (6) & (7) & (8) & (9) & (10) & (11) & (12) \\
\hline & \multicolumn{12}{|c|}{ Panel A } \\
\hline & \multicolumn{4}{|c|}{ 2-Year Frequency } & \multicolumn{4}{|c|}{ 3-Year Frequency } & \multicolumn{4}{|c|}{ 4-Year Frequency } \\
\hline Variables & $\Delta \mathrm{GDP}$ & $\Delta \mathrm{GDP}$ & $\triangle \mathrm{LOAN}$ & $\triangle \mathrm{LOAN}$ & $\Delta \mathrm{GDP}$ & $\Delta \mathrm{GDP}$ & $\triangle \mathrm{LOAN}$ & $\triangle \mathrm{LOAN}$ & $\Delta \mathrm{GDP}$ & $\triangle \mathrm{GDP}$ & $\triangle \mathrm{LOAN}$ & $\triangle \mathrm{LOAN}$ \\
\hline$\Delta$ Local Land Price & $\begin{array}{l}0.0304 * * * \\
(0.00816)\end{array}$ & $\begin{array}{l}0.0186 * * \\
(0.00768)\end{array}$ & $\begin{array}{l}0.110 * * * \\
(0.0200)\end{array}$ & $\begin{array}{c}0.0825 * * * \\
(0.0197)\end{array}$ & $\begin{array}{c}0.0390 * * * \\
(0.00783)\end{array}$ & $\begin{array}{l}0.0225^{* *} \\
(0.00926)\end{array}$ & $\begin{array}{l}0.155^{* * *} \\
(0.0201)\end{array}$ & $\begin{array}{l}0.113^{* * *} \\
(0.0201)\end{array}$ & $\begin{array}{c}0.0524 * * * \\
(0.00746)\end{array}$ & $\begin{array}{c}0.0382 * * * \\
(0.0105)\end{array}$ & $\begin{array}{c}0.152 * * * \\
(0.0122)\end{array}$ & $\begin{array}{l}0.112 * * * \\
(0.0127)\end{array}$ \\
\hline \multirow{2}{*}{$\begin{array}{l}\text { City Bank Share }{ }^{\star} \Delta \text { City } \\
\text { Land Price }\end{array}$} & & $0.0953 * * *$ & & $0.224 * * *$ & & $0.0852 * * *$ & & $0.218 * * *$ & & $0.0625 * * *$ & & $0.177 * * *$ \\
\hline & & $(0.0144)$ & & $(0.0468)$ & & $(0.0176)$ & & $(0.0416)$ & & $(0.0205)$ & & $(0.0363)$ \\
\hline City Bank Share & & $\begin{array}{c}0.0393 \\
(0.0775)\end{array}$ & & $\begin{array}{l}0.0973 \\
(0.147)\end{array}$ & & $\begin{array}{c}0.141 \\
(0.196)\end{array}$ & & $\begin{array}{c}0.491 \\
(0.344)\end{array}$ & & $\begin{array}{c}0.198 \\
(0.293)\end{array}$ & & $\begin{array}{c}0.805 \\
(0.554)\end{array}$ \\
\hline Observations & 517 & 517 & 517 & 517 & 329 & 329 & 329 & 329 & 235 & 235 & 235 & 235 \\
\hline Number of prefectures & 47 & 47 & 47 & 47 & 47 & 47 & 47 & 47 & 47 & 47 & 47 & 47 \\
\hline R-squared & 0.810 & 0.818 & 0.888 & 0.894 & 0.859 & 0.867 & 0.862 & 0.880 & 0.862 & 0.867 & 0.866 & 0.882 \\
\hline \multirow[t]{3}{*}{ First Stage F Statistic } & & & & 23.49 & & & & 26.88 & & & & 24.17 \\
\hline & \multicolumn{12}{|c|}{ Panel B (IV, $2^{\text {nd }}$ stage) } \\
\hline & & & & $\Delta \mathrm{GDP}$ & & & & $\Delta \mathrm{GDP}$ & & & & $\Delta \mathrm{GDP}$ \\
\hline \multirow[t]{2}{*}{$\triangle \mathrm{LOAN}$} & & & & $0.425^{* * *}$ & & & & $0.391 * * *$ & & & & $0.354 * * *$ \\
\hline & & & & $(0.0713)$ & & & & $(0.0970)$ & & & & $(0.135)$ \\
\hline \multirow[t]{2}{*}{$\Delta$ Local Land Price } & & & & $-0.0166 *$ & & & & -0.0215 & & & & -0.00154 \\
\hline & & & & $(0.00999)$ & & & & $(0.0181)$ & & & & $(0.0238)$ \\
\hline \multirow[t]{2}{*}{ City Bank Share } & & & & -0.00209 & & & & -0.0509 & & & & -0.0868 \\
\hline & & & & $(0.0589)$ & & & & $(0.165)$ & & & & $(0.302)$ \\
\hline R-squared & & & & 0.728 & & & & 0.846 & & & & 0.849 \\
\hline
\end{tabular}

Standard errors in parentheses 


\section{Not for Publication}

\section{Table C3: Replicate Table 3, using all prefectures (including the six city prefectures and Hokkaido)}

The reported results are based upon the value of city bank share prevailing as of 1979 , which is kept constant for the entire sample period. $\triangle L O A N$ and $\triangle G D P$ represent growth of local loan and output, respectively. $\Delta$ Local Land Price is growth of local land price index and $\Delta$ City Land Price is growth of land price index for six major cities. City Bank Share is a ratio of the number of city bank branches to the total number of bank branches in a given prefecture. Standard errors are adjusted for clustering within each prefecture. First stage F-statistics is Kleibergen-Paap rk Wald F statistic (weak identification test).

\begin{tabular}{|c|c|c|}
\hline & $(1)$ & $(2)$ \\
\hline & \multicolumn{2}{|c|}{ Panel A } \\
\hline & OLS & IV $\left(1^{\text {st }}\right.$ stage $)$ \\
\hline & $\Delta \mathrm{GDP}$ & $\triangle \mathrm{LOAN}$ \\
\hline \multirow[t]{2}{*}{$\Delta$ Local Land Price } & $0.00771^{* *}$ & $0.0321 *$ \\
\hline & $(0.00374)$ & $(0.0169)$ \\
\hline \multirow[t]{2}{*}{ City Bank Share* $\Delta$ City Land Price (1979) } & $0.105^{* * *}$ & $0.279 * * *$ \\
\hline & $(0.0126)$ & $(0.0563)$ \\
\hline Observations & 1081 & 1081 \\
\hline Number of prefectures & 47 & 47 \\
\hline \multirow[t]{5}{*}{ R-squared } & 0.724 & 0.876 \\
\hline & & 24.58 \\
\hline & & Panel B \\
\hline & & IV $\left(2^{\text {nd }}\right.$ stage $)$ \\
\hline & & $\Delta \mathrm{GDP}$ \\
\hline \multirow{2}{*}{$\triangle \mathrm{LOAN}$} & & $0.378 * * *$ \\
\hline & & $(0.0641)$ \\
\hline \multirow[t]{2}{*}{$\Delta$ Local Land Price } & & -0.00442 \\
\hline & & $(0.00528)$ \\
\hline R-squared & & 0.625 \\
\hline
\end{tabular}




\section{Not for Publication}

\section{Table C4: Replicate Table 4, using all prefectures (including the six city prefectures and Hokkaido)}

The table reports the results of the specification that separately estimate the effects of city land price growth on non-city bank lending (columns 1 and 2) and city bank lending (columns 3 and 4). Panels A and B in column 4 report the results of the first stage and second stage of an IV regression, respectively. $\triangle L O A N$ and $\triangle G D P$ represent growth of local loan and output, respectively. $\Delta$ Local Land Price is growth of local land price index and $\Delta$ City Land Price is growth of land

price index for six major cities. City Bank Share is a ratio of the number of city bank branches to the total number of bank branches in a given prefecture. Standard errors are adjusted for clustering within each prefecture. First stage F-statistics is Kleibergen-Paap rk Wald F statistic (weak identification test).

\begin{tabular}{|c|c|c|c|c|}
\hline & (1) & (2) & $\overline{(3)}$ & (4) \\
\hline & \multicolumn{4}{|c|}{ Panel A } \\
\hline & \multicolumn{2}{|c|}{$\triangle$ LOAN (Non-City Bank) } & \multicolumn{2}{|c|}{$\triangle \mathrm{LOAN}$ (City Bank) } \\
\hline$\Delta$ Local Land Price & $\begin{array}{c}0.0377 * \\
(0.0195)\end{array}$ & $\begin{array}{l}0.0331 * \\
(0.0194)\end{array}$ & $\begin{array}{c}0.0201^{* *} \\
(0.00759)\end{array}$ & $\begin{array}{c}0.00501 * * \\
(0.00209)\end{array}$ \\
\hline City Bank Share $* \Delta$ City Land Price & & $\begin{array}{c}0.0781 \\
(0.0515)\end{array}$ & & $\begin{array}{c}0.254 * * * \\
(0.0216)\end{array}$ \\
\hline City Bank Share & & $\begin{array}{c}0.0386 \\
(0.0668)\end{array}$ & & $\begin{array}{l}0.00701 \\
(0.0257)\end{array}$ \\
\hline Observations & 1077 & 1077 & 1077 & 1077 \\
\hline Number of prefectures & 47 & 47 & 47 & 47 \\
\hline R-squared & 0.860 & 0.860 & 0.277 & 0.485 \\
\hline First Stage F Statistic & & & & 138.5 \\
\hline & & & & $\begin{array}{c}\text { Panel B } \\
\text { (IV, } 2^{\text {nd }} \text { stage) } \\
\Delta \text { GDP }\end{array}$ \\
\hline$\triangle$ LOAN (City Banks) & & & & $\begin{array}{c}0.402 * * * \\
(0.0468)\end{array}$ \\
\hline$\Delta$ Local Land Price & & & & $\begin{array}{c}0.00609 * \\
(0.00340)\end{array}$ \\
\hline City Bank Share & & & & $\begin{array}{c}0.0182 \\
(0.0193)\end{array}$ \\
\hline R-squared & & & & 0.703 \\
\hline
\end{tabular}

\footnotetext{
${ }^{* * *} \mathrm{p}<0.01,{ }^{* *} \mathrm{p}<0.05,{ }^{*} \mathrm{p}<0.1$

Standard errors in parentheses
} 


\section{Not for Publication}

\section{Table C5: Replicate Table 5, using all prefectures (including the six city prefectures and Hokkaido)}

The table shows the results of specifications that control for differential correlation between local economies and cities. We compute the difference in income per capita (in absolute value) between each prefecture and cities (Income Diff) and then interact it to city land price growth and to city bank branch (columns 1-3). We interact the average distance to the cities (Distance) to city land price growth and to city bank branch share (columns 4-6). We also compute industry mix control (columns 7-9), which is constructed as: $\eta_{i t}=\gamma_{i j t-1} \eta_{j t}$ where $\eta_{i t}$ denotes the predicted output growth of prefecture $i$ in year $t$, $\gamma_{j i t-1}$ denote the (lagged) share of output in industry $j$ in prefecture $i$ in year $t-1$, and $\eta_{j t}$ denote the output growth of industry j in city prefectures in year $t$. Standard errors are adjusted for clustering within each prefecture.

\begin{tabular}{|c|c|c|c|c|c|c|c|c|c|}
\hline & (1) & (2) & (3) & (4) & (5) & (6) & (7) & (8) & (9) \\
\hline & $\begin{array}{l}\text { Reduced- } \\
\text { form } \\
\Delta \mathrm{GDP}\end{array}$ & $\begin{array}{c}\text { IV } \\
\text { (2nd stage) } \\
\Delta \mathrm{GDP}\end{array}$ & $\begin{array}{c}\text { IV } \\
\text { (1st stage) } \\
\Delta \text { LOAN }\end{array}$ & $\begin{array}{c}\text { Reduced- } \\
\text { form } \\
\Delta \mathrm{GDP}\end{array}$ & $\begin{array}{c}\text { IV } \\
\text { (2nd stage) } \\
\Delta \text { GDP }\end{array}$ & $\begin{array}{c}\text { IV } \\
\text { (1st stage) } \\
\Delta \text { LOAN }\end{array}$ & $\begin{array}{c}\text { Reduced- } \\
\text { form } \\
\Delta \mathrm{GDP}\end{array}$ & $\begin{array}{c}\text { IV } \\
\text { (2nd stage) } \\
\Delta \mathrm{GDP}\end{array}$ & $\begin{array}{c}\text { IV } \\
\text { (1st stage) } \\
\Delta \text { LOAN }\end{array}$ \\
\hline City Bank Share* $\Delta$ City Land Price & $\begin{array}{c}0.0866 * * * \\
(0.0177)\end{array}$ & & $\begin{array}{c}0.241^{* * *} \\
(0.0463)\end{array}$ & $\begin{array}{c}0.0702 * * * \\
(0.0169)\end{array}$ & & $\begin{array}{c}0.207 * * * \\
(0.0519)\end{array}$ & $\begin{array}{c}0.0626 * * * \\
(0.0153)\end{array}$ & & $\begin{array}{l}0.206 * * * \\
(0.0530)\end{array}$ \\
\hline$\triangle \mathrm{LOAN}$ & & $\begin{array}{c}0.360 * * * \\
(0.100)\end{array}$ & & & $\begin{array}{c}0.339 * * * \\
(0.110)\end{array}$ & & & $\begin{array}{c}0.304 * * * \\
(0.0919)\end{array}$ & \\
\hline$\Delta$ Local Land Price & $\begin{array}{l}0.00556 * \\
(0.00283)\end{array}$ & $\begin{array}{l}-0.00419 \\
(0.00565)\end{array}$ & $\begin{array}{c}0.0271 * \\
(0.0145)\end{array}$ & $\begin{array}{c}0.00583 * \\
(0.00293)\end{array}$ & $\begin{array}{c}-0.00364 \\
(0.00572)\end{array}$ & $\begin{array}{l}0.0279 * \\
(0.0147)\end{array}$ & $\begin{array}{l}0.00574 * \\
(0.00293)\end{array}$ & $\begin{array}{l}-0.00275 \\
(0.00497)\end{array}$ & $\begin{array}{l}0.0279 * \\
(0.0147)\end{array}$ \\
\hline City Bank Share & $\begin{array}{l}0.0517 * \\
(0.0294)\end{array}$ & $\begin{array}{c}0.0212 \\
(0.0324)\end{array}$ & $\begin{array}{c}0.0847 \\
(0.0771)\end{array}$ & $\begin{array}{c}0.137 * \\
(0.0806)\end{array}$ & $\begin{array}{c}0.0116 \\
(0.0999)\end{array}$ & $\begin{array}{c}0.369 * * \\
(0.147)\end{array}$ & $\begin{array}{c}0.131 * \\
(0.0766)\end{array}$ & $\begin{array}{c}0.0188 \\
(0.0919)\end{array}$ & $\begin{array}{c}0.368 * * \\
(0.147)\end{array}$ \\
\hline Income Diff* $\Delta$ City Land Price & $\begin{array}{c}-0.0217^{* * *} \\
(0.00545)\end{array}$ & $\begin{array}{l}-0.00279 \\
(0.00837)\end{array}$ & $\begin{array}{c}-0.0527 * * * \\
(0.0167)\end{array}$ & $\begin{array}{c}-0.0136 * * \\
(0.00608)\end{array}$ & $\begin{array}{r}-0.000878 \\
(0.00781)\end{array}$ & $\begin{array}{c}-0.0375 * \\
(0.0190)\end{array}$ & $\begin{array}{c}-0.0117^{*} \\
(0.00600)\end{array}$ & $\begin{array}{l}-0.000386 \\
(0.00687)\end{array}$ & $\begin{array}{l}-0.0372 * \\
(0.0190)\end{array}$ \\
\hline Income Diff*City Bank Share & $\begin{array}{c}-0.0279 * * \\
(0.0111)\end{array}$ & $\begin{array}{c}-0.00756 \\
(0.0192)\end{array}$ & $\begin{array}{c}-0.0566 * * * \\
(0.0172)\end{array}$ & $\begin{array}{c}-0.0295^{* * *} \\
(0.0103)\end{array}$ & $\begin{array}{l}-0.00774 \\
(0.0203)\end{array}$ & $\begin{array}{c}-0.0641 * * * \\
(0.0223)\end{array}$ & $\begin{array}{c}-0.0300 * * * \\
(0.00986)\end{array}$ & $\begin{array}{l}-0.0105 \\
(0.0181)\end{array}$ & $\begin{array}{c}-0.0642^{* * *} \\
(0.0224)\end{array}$ \\
\hline Distance $* \Delta$ City Land Price & & & & $\begin{array}{l}-0.0270 * \\
(0.0154)\end{array}$ & $\begin{array}{l}-0.0126 \\
(0.0125)\end{array}$ & $\begin{array}{c}-0.0423 \\
(0.0436)\end{array}$ & $\begin{array}{c}-0.0252 * \\
(0.0142)\end{array}$ & $\begin{array}{c}-0.0124 \\
(0.0117)\end{array}$ & $\begin{array}{l}-0.0420 \\
(0.0432)\end{array}$ \\
\hline Distance*City Bank Share & & & & $\begin{array}{c}-0.105 \\
(0.0781)\end{array}$ & $\begin{array}{c}0.0161 \\
(0.0901)\end{array}$ & $\begin{array}{c}-0.356^{* *} \\
(0.140)\end{array}$ & $\begin{array}{c}-0.0969 \\
(0.0741)\end{array}$ & $\begin{array}{c}0.0109 \\
(0.0838)\end{array}$ & $\begin{array}{c}-0.355^{* *} \\
(0.140)\end{array}$ \\
\hline Industry Mix Control $\left(\eta_{i t}\right)$ & & & & & & & $\begin{array}{l}0.525^{*} \\
(0.274)\end{array}$ & $\begin{array}{l}0.501^{*} \\
(0.267)\end{array}$ & $\begin{array}{l}0.0789 \\
(0.216)\end{array}$ \\
\hline Observations & 1081 & 1081 & 1081 & 1081 & 1081 & 1081 & 1081 & 1081 & 1081 \\
\hline R-squared & 0.728 & 0.636 & 0.878 & 0.729 & 0.648 & 0.879 & 0.733 & 0.670 & 0.879 \\
\hline Number of prefectures & 47 & 47 & 47 & 47 & 47 & 47 & 47 & 47 & 47 \\
\hline First Stage F Statistic & & 27.07 & & & 15.91 & & & 15.10 & \\
\hline
\end{tabular}

$* * * \mathrm{p}<0.01, * * \mathrm{p}<0.05, * \mathrm{p}<0.1$

Standard errors in parentheses 


\section{Not for Publication}

Table C7: Replicate Table 10, using all prefectures (including the six city prefectures and Hokkaido)

Columns 1-2 report the results of panel corrected standard errors (Beck and Katz, 1995). Columns 3-4 report the results of Driscoll-Kraay standard errors (Driscoll and Kraay, 1998).Columns 3-4 report the results of two-way clustering (Petersen, 2009; Cameron, Gelbach, and Miller, 2006).

\begin{tabular}{|c|c|c|c|c|c|c|}
\hline & (1) & (2) & (3) & (4) & (5) & (6) \\
\hline & \multicolumn{2}{|c|}{$\begin{array}{c}\text { Panel-Corrected Standard } \\
\text { Errors }\end{array}$} & \multicolumn{2}{|c|}{$\begin{array}{c}\text { Driscoll-Kraay Standard } \\
\text { Errors }\end{array}$} & \multicolumn{2}{|c|}{$\begin{array}{l}\text { Petersen-Cameron-Gelbach- } \\
\text { Miller Two-way Clustering }\end{array}$} \\
\hline & $\triangle \mathrm{LOAN}$ & $\Delta \mathrm{GDP}$ & $\triangle \mathrm{LOAN}$ & $\Delta \mathrm{GDP}$ & $\triangle \mathrm{LOAN}$ & $\Delta \mathrm{GDP}$ \\
\hline City Bank Share* $\Delta$ City Land Price & $\begin{array}{c}0.206^{* *} \\
(0.103)\end{array}$ & $\begin{array}{c}0.0626 * * * \\
(0.0230)\end{array}$ & $\begin{array}{l}0.206^{* *} \\
(0.0835)\end{array}$ & $\begin{array}{c}0.0626 * * * \\
(0.0198)\end{array}$ & $\begin{array}{l}0.206 * * * \\
(0.0542)\end{array}$ & $\begin{array}{c}0.0626 * * * \\
(0.0157)\end{array}$ \\
\hline$\Delta$ Local Land Price & $\begin{array}{l}0.0279 * * \\
(0.0118)\end{array}$ & $\begin{array}{c}0.00574 \\
(0.00394)\end{array}$ & $\begin{array}{l}0.0279 * \\
(0.0143)\end{array}$ & $\begin{array}{l}0.00574 * \\
(0.00338)\end{array}$ & $\begin{array}{l}0.0279 * \\
(0.0150)\end{array}$ & $\begin{array}{l}0.00574 * \\
(0.00299)\end{array}$ \\
\hline City Bank Share & $\begin{array}{c}0.368 \\
(0.309)\end{array}$ & $\begin{array}{c}0.131 \\
(0.0974)\end{array}$ & $\begin{array}{c}0.368^{* * *} \\
(0.119)\end{array}$ & $\begin{array}{c}0.131 \\
(0.0827)\end{array}$ & $\begin{array}{l}0.368^{* *} \\
(0.150)\end{array}$ & $\begin{array}{c}0.131 \\
(0.0784)\end{array}$ \\
\hline Income Diff* $\Delta$ City Land Price & $\begin{array}{c}-0.0372 * * * \\
(0.0111)\end{array}$ & $\begin{array}{c}-0.0117 \\
(0.00950)\end{array}$ & $\begin{array}{c}-0.0372^{* * *} \\
(0.00983)\end{array}$ & $\begin{array}{l}-0.0117 \\
(0.0100)\end{array}$ & $\begin{array}{l}-0.0372 * \\
(0.0194)\end{array}$ & $\begin{array}{l}-0.0117^{*} \\
(0.00613)\end{array}$ \\
\hline Income Diff*City Bank Share & $\begin{array}{l}-0.0642 \\
(0.0536)\end{array}$ & $\begin{array}{c}-0.0300 * * * \\
(0.00962)\end{array}$ & $\begin{array}{c}-0.0642 * * * \\
(0.0170)\end{array}$ & $\begin{array}{c}-0.0300 * * * \\
(0.00844)\end{array}$ & $\begin{array}{c}-0.0642 * * * \\
(0.0229)\end{array}$ & $\begin{array}{l}-0.0300 * * * \\
(0.0101)\end{array}$ \\
\hline Distance $* \Delta$ City GDP & $\begin{array}{l}-0.0420 \\
(0.0287)\end{array}$ & $\begin{array}{l}-0.0252 \\
(0.0177)\end{array}$ & $\begin{array}{l}-0.0420^{*} \\
(0.0217)\end{array}$ & $\begin{array}{l}-0.0252 * * \\
(0.00973)\end{array}$ & $\begin{array}{l}-0.0420 \\
(0.0442)\end{array}$ & $\begin{array}{l}-0.0252^{*} \\
(0.0146)\end{array}$ \\
\hline Distance*City Bank Share & $\begin{array}{l}-0.355 \\
(0.330)\end{array}$ & $\begin{array}{l}-0.0969 \\
(0.104)\end{array}$ & $\begin{array}{c}-0.355^{* * *} \\
(0.107)\end{array}$ & $\begin{array}{l}-0.0969 \\
(0.0890)\end{array}$ & $\begin{array}{c}-0.355^{* *} \\
(0.144)\end{array}$ & $\begin{array}{l}-0.0969 \\
(0.0758)\end{array}$ \\
\hline Industry Mix Control $\left(\eta_{i t}\right)$ & $\begin{array}{l}0.0789 \\
(0.525)\end{array}$ & $\begin{array}{l}0.525^{* *} \\
(0.206)\end{array}$ & $\begin{array}{c}0.0789 \\
(0.378)\end{array}$ & $\begin{array}{c}0.525 \\
(0.363)\end{array}$ & $\begin{array}{c}0.0789 \\
(0.221)\end{array}$ & $\begin{array}{l}0.525 * \\
(0.280)\end{array}$ \\
\hline Observations & 1081 & 1081 & 1081 & 1081 & 1081 & 1081 \\
\hline Number of prefectures & 47 & 47 & 47 & 47 & 47 & 47 \\
\hline R-squared & 0.880 & 0.736 & 0.880 & 0.736 & 0.880 & 0.736 \\
\hline
\end{tabular}

Standard errors in parentheses 